\title{
CONGRESO DE INICIACIÓN CIENTÍFICA Y PROFESIONAL DE LOS ESTUDIANTES DE LA UNIVERSIDAD DE MAGALLANES-CICYPE 2009
}

\author{
CONGRESS OF SCIENTIFIC AND PROFESSIONAL INITIATION OF STUDENTS \\ FROM THE UNIVERSIDAD DE MAGALLANES CICYPE 2009
}

Ingrid Hebel ${ }^{1} \&$ Mirna Pizarro²

El Congreso de Iniciación Científica y Profesional de los Estudiantes de la Universidad de Magallanes-CICYPE, es una actividad que se ha llevado a cabo desde el año 2002. Desde entonces, este congreso ha permitido promover la actividad científica en un importante sector de la población de estudiantes de todas las disciplinas y especialidades dentro de nuestra universidad.

Dado que se necesitan profesionales que se atrevan a asumir la realidad desde diversas ópticas y puntos de vista de las distintas disciplinas, este congreso ha fortalecido la participación de los estudiantes en la actividad científica como una estrategia importante para la formación académica. Los alumnos participantes han demostrado un gran compromiso y responsabilidad con la formación de capacidades en investigación y el intercambio de experiencias. La formación científica supone-además-el compromiso con el saber pensar y ello está en el centro de la ciudadanía. Hacer ciencia es también hacer historia, por lo tanto, el desarrollo de la actividad científica permite ser protagonistas de dicha historia y ser personas activas y no espectadoras frente a los desafíos que impone la realidad.
Por otro lado, este evento ha propiciado el encuentro de estudiantes y docentes de la universidad para el desarrollo de la actividad científica y el trabajo en equipos multidisciplinarios. La participación de los estudiantes ha mostrado importantes evidencias de la producción científica en nuestra universidad, y compromiso con el desarrollo de la ciencia en la región, participando alumnos que se encuentran desarrollando trabajos o proyectos de investigación, trabajos de titulación o tesis en su especialidad.

Por todo lo anterior, el congreso ha ofrecido un espacio para fortalecer la participación de estudiantes no sólo en la actividad científica, sino que también ha favorecido la interrelación del alumnado en sus diferentes áreas de investigación.

Aunque se consideran para este congreso todas las áreas temáticas que imparte la universidad, el año 2009 se presentaron trabajos de investigación en las áreas de la Tecnología y Ciencias de la Ingeniería, Ciencias Sociales y de la Comunicación, Ecología y Ciencias Ambientales, Tecnologías y Ciencias Silvoagropecuarias y Ciencias Básicas.

$1 \quad$ Universidad de Magallanes, Facultad de Ciencias, Punta Arenas, Chile. ingrid.hebel@umag.cl

2 Universidad de Magallanes, Facultad de Humanidades Ciencias Sociales y de la Salud, Punta Arenas, Chile. mirna.pizarro@ umag.cl 


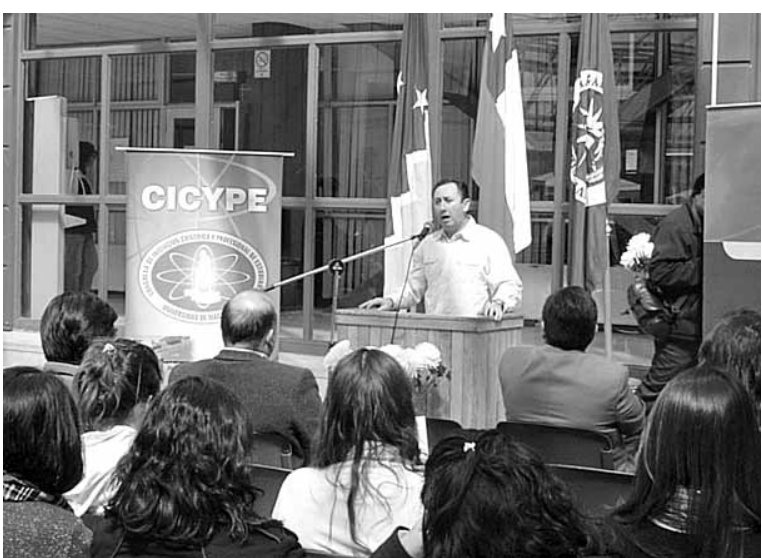

Fig. 1. Ceremonia de Inauguración CICYPE 2009. En la foto, Dr. Andrés Mansilla, Director de Investigación y Postgrado.

Para la evaluación de los trabajos contamos con una comisión integrada por representantes de diversas Facultades, quienes debieron asumir la responsabilidad de deliberar bajo criterios como: calidad, precisión y creatividad del estudio; además, de dominio del contenido y calidad de la presentación del trabajo, para elegir los tres mejores trabajos en cada modalidad (poster y presentación oral). De la misma evaluación, se eligieron los mejores trabajos en el tema cambio climático.

En general, se presentaron un total de 28 trabajos en modalidad de exposición oral y 14 trabajos en categoría poster. En categoría oral tuvieron un total de 15 minutos para su exposición y 5 minutos para preguntas. En categoría poster los alumnos tuvieron 10 minutos para exponer sus trabajos. Participaron alrededor de 75 alumnos.

La cantidad de trabajos enviados para este evento evidencia una alta motivación con la actividad científica y el compromiso de los docentes, que se traduce en el apoyo que ellos brindan a sus alumnos para que participen. Hay que destacar también, que todos los trabajos presentados se refieren a temas de interés regional.

Cerca de 40 profesores compartieron las tareas tanto en los preparativos del congreso, como en la evaluación de trabajos y en el apoyo científico para el desarrollo de los trabajos de los alumnos. Participaron profesores de la Universidad de Magallanes, del Instituto Antártico Chileno (INACH) y del Centro de Estudios del Cuaternario (CEQUA).

En su quinta versión, se consideró como actividad paralela una sesión especial para trabajos

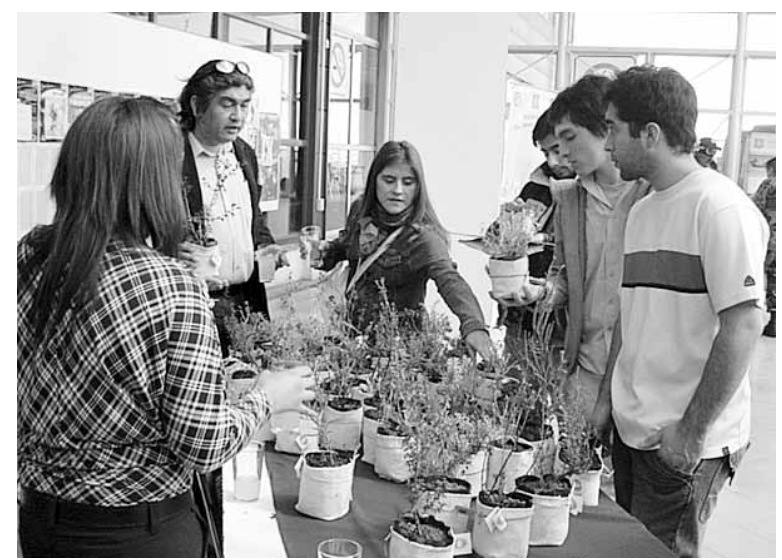

Fig. 2. Plantas de flora nativa entregadas a los alumnos como símbolo del congreso durante la Inauguración del CICYPE 2009.

sobre la temática cambio climático, debido a que este evento se realizó en forma excepcional en conjunto con el Coloquio sobre Cambio Climático: Climate Change in Magellan and Antarctic Regions: Evidence and Challenge for the Future. Los mejores trabajos sobre este tema fueron presentados y premiados en sesión especial de clausura del Coloquio Internacional sobre cambio climático, el día viernes 30 de octubre. Durante la ceremonia, las cuatro alumnas ganadoras pudieron exponer sus trabajos tanto a los expositores, destacados investigadores de origen nacional e internacional que asistieron al coloquio, como a la comunidad en general.

Los mejores trabajos sobre Cambio Climático fueron los siguientes:

¿Qué podemos hacer para enfrentar el cambio climático desde la educación preescolar?

En este proyecto de Paulina Mandujano y Verónica Vergara, de Educación Parvularia, exponen su preocupación por la falta de un comportamiento y actitudes de las personas en favor del medio ambiente, especialmente considerando la inquietud a nivel mundial, nacional y regional por los constantes cambios que sufre el planeta a causa del cambio climático. En ese sentido, se planteó un proyecto con el fin de educar hacia la conciencia del problema del cambio climático e involucrar a los párvulos en la búsqueda de soluciones y en la adquisición de hábitos y formación de actitudes de respeto hacia el medio ambiente. 
Estudio del impacto de la erupción del volcán Chaitén como contaminante del medio ambiente patagónico y antártico

En este estudio, Carolina Toro, estudiante de la carrera de Biología Marina, determina la composición química de la precipitación nevosa colectada en la Antártica y Patagonia, estableciendo la posible influencia de las cenizas provenientes del volcán Chaitén sobre la composición química de ésta y su impacto ambiental, a través del aporte de metales, en ambientes marinos australes, producto de la gran cantidad de partículas y gases que fueron inyectados al interior de la estratósfera en la reciente erupción protagonizada por el volcán Chaitén el 2 de mayo de 2008. Los resultados obtenidos no solo aportarían información crucial para comprender los efectos de fenómenos geológicos sobre el medio ambiente austral, sino que también para el entendimiento de posibles cambios en el medio ambiente a nivel global.

Estudio preliminar de las concentraciones de metales pesados (cd, cu, cr, pb, $v$ y zn) en zona costera de bahía Fildes, isla Rey Jorge, Península Antártica Chilena

Este proyecto de Ninoska Ocampo, de la carrera de Biología Marina, tiene por objetivo proveer información preliminar sobre la bioconcentración de metales pesados en organismos marinos y la relación directa que existe con la biota que los rodea estudiando un área determinada de Bahía Fildes, Isla Rey Jorge. Esto tiene por finalidad evaluar la biodisponibilidad de los metales en el área, dado que la contaminación por metales pesados de los ambientes costeros reciben una gran polución proveniente de áreas urbanizadas, vertederos industriales y ríos, volviéndose grave la situación por la capacidad acumulativa que presentan los organismos.

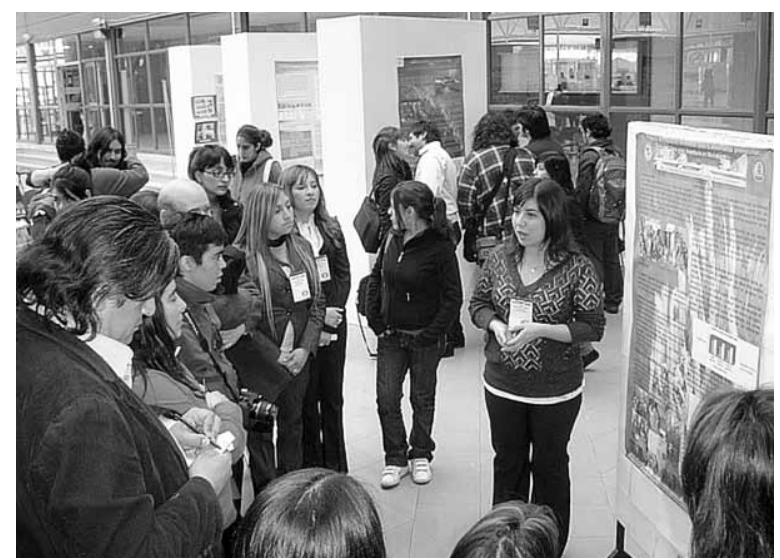

Fig. 3. Presentación en modalidad Poster del Proyecto de Educación Preescolar sobre Biodiversidad del Cabo de Hornos.

Finalmente, el introducir e integrar la investigación en la formación de pregrado despierta la vocación científica y con esto se incentiva los potenciales talentos en pregrado. Estos valores del congreso abrigan la esperanza de que entre los estudiantes que participan, puedan encontrarse futuros académicos, investigadores y, por supuesto, futuros profesionales preparados para nuevos y exigentes escenarios. La idea de generar un espacio de encuentro profesional y científico entre todos los alumnos de la Universidad organizado y desarrollado en Punta Arenas, potencia las capacidades de los estudiantes al emular un encuentro de alto nivel como en los grandes eventos científicos, dándole una posibilidad nueva de formación a aquellos estudiantes que no les resulta fácil participar en congresos en otras zonas del país.

Así, el Congreso de Iniciación Científica se convierte en un evento de gran importancia para el estudiante de la Universidad de Magallanes, ya que los alumnos adquieren experiencia que les servirá en estudios de postgrado y en su vida profesional. Es un estímulo que contribuye a mejorar su análisis crítico, lograr madurez intelectual y la comprensión de la ciencia. 
